\title{
Angle-dependent strong-field ionization and fragmentation of carbon dioxide measured using rotational wave packets
}

DOI:

10.1103/PhysRevA.102.043119

\section{Document Version}

Accepted author manuscript

Link to publication record in Manchester Research Explorer

\section{Citation for published version (APA):}

Lam, H. V. S., Yarlagadda, S., Venkatachalam, A., Wangjam, T. N., Kushawaha, R. K., Cheng, C., Svihra, P., Nomerotski, A., Weinacht, T., Rolles, D., \& Kumarappan, V. (2020). Angle-dependent strong-field ionization and fragmentation of carbon dioxide measured using rotational wave packets. Physical Review A, 102(4), [043119]. https://doi.org/10.1103/PhysRevA.102.043119

Published in:

Physical Review A

\section{Citing this paper}

Please note that where the full-text provided on Manchester Research Explorer is the Author Accepted Manuscript or Proof version this may differ from the final Published version. If citing, it is advised that you check and use the publisher's definitive version.

\section{General rights}

Copyright and moral rights for the publications made accessible in the Research Explorer are retained by the authors and/or other copyright owners and it is a condition of accessing publications that users recognise and abide by the legal requirements associated with these rights.

\section{Takedown policy}

If you believe that this document breaches copyright please refer to the University of Manchester's Takedown Procedures [http://man.ac.uk/04Y6Bo] or contact uml.scholarlycommunications@manchester.ac.uk providing relevant details, so we can investigate your claim.

\section{OPEN ACCESS}




\title{
Angle-dependent strong-field ionization and fragmentation of carbon dioxide measured using rotational wave-packets
}

\author{
Huynh Lam, ${ }^{1}$ Suresh Yarlagadda, ${ }^{1}$ Anbu Venkatachalam, ${ }^{1}$ Tomthin Nganba Wangjam, ${ }^{1}$ Rajesh K. Kushawaha, ${ }^{1}$ \\ Chuan Cheng, ${ }^{2}$ Peter Svihra, ${ }^{3,4}$ Andrei Nomerotski, ${ }^{5}$ Thomas Weinacht, ${ }^{2}$ Daniel Rolles, ${ }^{1}$ and Vinod Kumarappan ${ }^{1}$ \\ ${ }^{1}$ J. R. Macdonald Laboratory, Kansas State University, Manhattan, KS 66506, USA \\ ${ }^{2}$ Department of Physics and Astronomy, Stony Brook University, Stony Brook, New York 11794, USA \\ ${ }^{3}$ Department of Physics, Faculty of Nuclear Sciences and Physical Engineering, Czech Technical University, Prague 115 19, CZ \\ ${ }^{4}$ Department of Physics and Astronomy, School of Natural Sciences, \\ University of Manchester, Manchester M13 9PL, UK \\ ${ }^{5}$ Brookhaven National Laboratory, Upton, New York 11793, USA
}

(Dated: May 4, 2020)

\begin{abstract}
In this work, we experimentally study the angle-dependent single ionization of carbon dioxide $\left(\mathrm{CO}_{2}\right)$ by linearly and circularly polarized pulses. The angle dependence of the ionization probability by linearly polarized pulses extracted from time-domain measurements on an impulsively-excited rotational wave-packet is compared with data obtained from a direct angle-scan measurement. The results from the measurement with linear and circular polarization are consistent with the adiabatic ionization approximation. We extend the time-domain method to extract the dependence of the asymptotic momentum distribution of fragment ions on the orientation of the molecular axis, and apply it to investigate dissociative double ionization of $\mathrm{CO}_{2}$. We show that such measurements can directly test the validity of the axial recoil approximation.
\end{abstract}

PACS numbers: 37.10.Vz, 33.15.Bh, 33.80.-b,33.20.Sn,33.80.Rv,33.80.Wz

\section{INTRODUCTION}

Ionization is the first step in many high-order strongfield processes, including high-harmonic generation, above-threshold ionization and laser-induced electron diffraction [1-8]. However, strong-field ionization (SFI) of molecules is still not well understood. Although there are several models [9-11] that provide accurate intensity dependent or total ionization ionization yields from atoms at infrared wavelengths, molecular ionization models are still being developed and face many difficulties [5, 12-16]. For example, the measured angle-dependent single ionization of $\mathrm{N}_{2}$ and $\mathrm{O}_{2}$ can be reproduced by theoretical models [17-19] while that is not the case for $\mathrm{CO}_{2}$. The angular width of the angle-dependent single ionization of $\mathrm{CO}_{2}$ reported by Pavičić et al. [17] appears to be too narrow, with the problem suspected to be in the deconvolution process. Later measurements at different intensity or wavelength were done by Thomann et al. [20], Weber et al. [21] and the topic is still being discussed actively [17, 19-33].

In this work, we revisit this problem using a timedomain approach we call Orientation Resolution through Rotational Coherence Spectroscopy (ORRCS) [34] to retrieve the angle-dependent ionization probability of $\mathrm{CO}_{2}$ with significantly better angular resolution. We launch a rotational wave-packet created by 1D impulsive alignment using a linearly-polarized non-resonant laser pulse $[35,36]$ and measure the molecular ion yield as a function of delay between the aligning pump and the ionizing probe pulse. We then use a fitting procedure to retrieve the angle-dependent ionization yield. Our goal is to determine this dependence with substantially higher angular resolution than prior measurements $[17,20,21]$ by using cold molecules and multi-pulse alignment. The retrieved angle dependence is compared with data obtained from a direct angle-scan measurement, and checked for consistency with results from measurements using circularly polarized ionizing probe pulse through a geometrical transformation. This easy adaptation of the time-domain approach to different ellipticity of laser pulses can be used to explore other dynamics, especially processes triggered by re-collision $[4,13,21,37,38]$.

We further extend this time-domain approach to photo-ion momentum measurements, where the axial recoil approximation is often used without validation. In order to test the validity of the approximation, it is necessary to measure the molecular axis distribution independently. We show that this can be achieved by measuring the momentum distributions of fragments in a pumpprobe experiment in which the molecules are impulsively aligned by a non-resonant pump and then dissociated by a probe pulse. The delay-dependent momentum distributions are analyzed to generate a $2 \mathrm{D}$ probability distribution function (polar angle of the fragment momentum vector and orientation of the molecular axis in the laboratory frame) that directly reflects the validity of the axial recoil approximation.

\section{EXPERIMENTAL SETUP}

A schematic diagram of the experimental setup is shown in Fig. 1. Pulses from the Kansas Light Source (KLS) laser $(2 \mathrm{~mJ} /$ pulse, $785 \mathrm{~nm}$ center wavelength, 35 fs pulse duration, $2 \mathrm{kHz}$ repetition rate) are split into two with a broadband $75 \%$-reflection beam-splitter. The transmitted pulse is expanded by a telescope and serves 
as the probe. The reflected pulse is further split into two alignment pulses, stretched by SF-11 glass to about 100 fs and 250 fs pulses and down-collimated by another telescope. The compressor grating was optimized for the shortest probe pulses. The duration of the pulses is measured using a FROG [39] before the beams enter the vacuum chamber. A window was added to correct for going through the chamber window. The focal spot size of the two pump beams is about 2 times larger than that of the probe beam, which allows us to measure signal only from well-aligned molecules, and reduce the effect of averaging over the distribution of pump intensities in the focus. Achromatic zero-order half-wave plates and cube polarizers in both beams allow independent control of power. Circularly polarized pulses are made by adding achromatic zero-order quarter-wave plates to both the beams and characterized by measuring the Stokes polarization parameters [40]. The delay between the aligning pulses and the probe pulse is varied using a computer-controlled translation stage. The beams are then back-focused inside a vacuum chamber by a $25 \mathrm{~cm}$ focal length concave mirror before they interact with rotationally cold molecules $\left(<3 \mathrm{~K}\right.$ for $\left.\mathrm{CO}_{2}\right)$ produced by supersonic expansion $\left(0.5 \% \mathrm{CO}_{2}\right.$ in He at a total pressure of 70 bar) through a $1 \mathrm{kHz}$ Even-Lavie valve [41].

The 100 fs aligning pulse is delayed by 32.25 ps with respect to the $250 \mathrm{fs}$ aligning pulse. This value corresponds to the time when the molecular sample reaches the highest alignment created by the first pulse $(3 / 4$-th revival of $\mathrm{CO}_{2}$ ). Some experiments were performed with only one aligning pulse.

The aligning pulses are mechanically chopped at 500 $\mathrm{Hz}$, letting two consecutive laser pulses through and blocking the next two consecutive laser pulses in each cycle. Since the Even-Lavie valve operates at $1 \mathrm{kHz}$, we get four combinations of pump and gas (the probe is always present) pulses in each chopper cycle: pump-probegas, pump-probe-no gas, probe-gas and probe-no gas. A velocity map imaging (VMI) spectrometer [42] using a thick-lens design [43] with micro-channel plate detector (MCP) and fast phosphor screen was used in Time-ofFlight (ToF) mode for measuring ion yields, or in imaging mode with the images captured by a Tpx3Cam detector $[44,45]$ in order to determine the angular distribution of fragment ions.

The signal was measured as a function of pump-probe delay for each configuration of pump-probe-gas trigger status (details of the synchronization with Tpx3Cam detector are provided in section IV B). At each delay, the background signal measured without the jet on is subtracted from the signal measured with the jet on, and then normalized to the background-corrected signal from the unaligned gas. The corrected ion yield $Y(t)$ used in further analysis is

$$
Y(t)=\frac{[\text { Pump, Probe, Gas }]-[\text { Pump, Probe }]}{[\text { Probe, Gas }]-[\text { Probe }]}
$$

Delay scans are repeated multiple times and averaged.

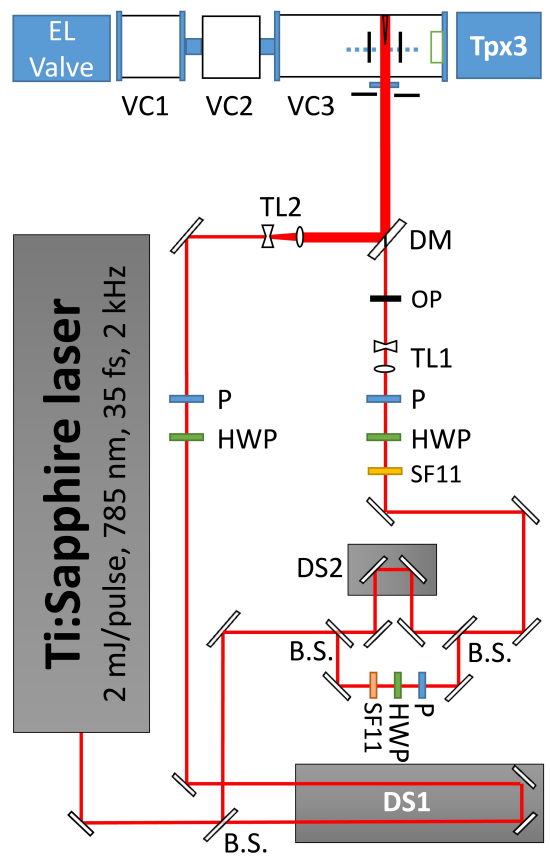

FIG. 1. Experimental setup. B.S. = beam splitter, DS1 = Motorized delay stage, DS2 = Manual delay stage, $\mathrm{P}=$ Polarizer, HWP = Half-waveplate, SF11 = SF11 glass, TL1 = Shrinking telescope, $\mathrm{OP}=$ Optical chopper running at 500 $\mathrm{Hz}, \mathrm{TL} 2=$ Expanding telescope, $\mathrm{DM}=$ Drilled mirror with a hole through the center, EL Valve = Even-Lavie valve running at $1 \mathrm{kHz}, \mathrm{Tpx} 3=\mathrm{Tpx} 3$ time-stamping pixel detector, $\mathrm{VC} 1=$ vacuum gas jet chamber $\left(10^{-5}\right.$ Torr $), \mathrm{VC} 2=$ middle chamber to improve differential pumping $\left(10^{-8}\right.$ Torr $)$, VC3 = VMI chamber $\left(10^{-10}\right.$ Torr $)$.

This procedure corrects for both short and long term fluctuations in the gas density and averages over any drifts in pump-probe overlap.

In the momentum measurements, the background count rate for fragment ions was small compared to the rate with the jet. Moreover, negative values introduced by subtraction of images complicate the interpretation of the result, so we did not subtract background images. Instead, the VMI images $M_{2 D}\left(\vec{k}_{2 D}, t\right)$ were normalized to the total count from the unaligned gas measurements to correct for the fluctuation in gas density and laser intensity as expressed in Eq. (2).

$$
M_{2 D}\left(\vec{k}_{2 D}, t\right)=\frac{[\text { Pump, } \text { Probe, Gas }]}{\text { Count [Probe, Gas }]}
$$

We discuss the data and analysis of the molecular ion yield data in Sec. III, and the momentum measurements in Sec. IV. 


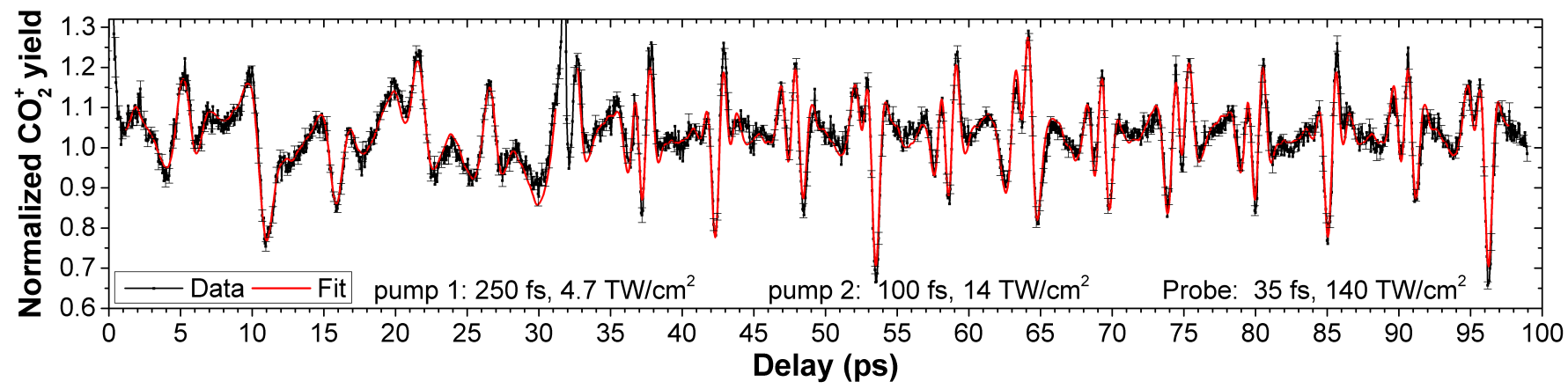

FIG. 2. Experimental data and fit to the $\mathrm{CO}_{2}^{+}$ionization yield obtained with a linearly polarized probe as function of delay. The pulse duration and intensity of the first and second aligning pulses are $250 \mathrm{fs}, 4.7 \mathrm{TW} / \mathrm{cm}^{2}$ and $100 \mathrm{fs}, 14 \mathrm{TW} / \mathrm{cm}^{2}$, respectively. The $100 \mathrm{fs}$ aligning pulse is delayed by $32.25 \mathrm{ps}$ with respect to the $250 \mathrm{fs}$ aligning pulse $\left(3 / 4\right.$ revival of $\left.\mathrm{CO}_{2}\right)$. The ionizing pulse has 35 fs pulse width and $140 \mathrm{TW} / \mathrm{cm}^{2}$ intensity. textcolorblueWe measured the focal spot size of the beam using a camera and the pulse duration using a FROG. Assume that the pulse has the Gaussian profile in both space and time, the intensity of the probe pulse can be estimated. All pulses are linearly polarized in the same direction.

\section{ANGLE DEPENDENCE OF STRONG-FIELD SINGLE IONIZATION OF $\mathrm{CO}_{2}$}

\section{A. Time-domain approach using ORRCS}

The angle-dependent ionization probability can be obtained from an angle-scan measurement in which the ionization yield $Y(\alpha)$ is measured as a function of angle $\alpha$ between the polarization axes of the aligning pump pulse and the ionizing probe pulse $[17,21,46,47]$, or from a delay-scan measurement in which the ionization yield $Y(t)$ is measured as a function of pump-probe delay between a pump pulse used for impulsive alignment and a probe pulse for ionization $[14,20,34,48]$. In both cases, a deconvolution process needs to be conducted in order to separate the distribution of the molecular axis from the laboratory frame (LF) data and extract the angle dependence of ionization in the molecular frame (MF) $[1,14,17,20,21,34,46-50]$.

The time-domain approach has been demonstrated with different types of molecules (linear, symmetricand asymmetric-top) [1, 14, 34, 49-51]. In particular, Makhija et al. [34] have shown that both the rotational wave-packet and the angle-dependence of ionization can be retrieved from the delay dependent data under the assumption that rigid-rotor TDSE calculations accurately describe the rotational wave-packet. For asymmetric top molecules, this approach provides access to the dependence of ionization on two Euler angles. Following is a brief summary of the method, named ORRCS, as it applies to linear molecules.

The measurement $Y(t)$ in the LF is a convolution of the MF angle-dependent signal $R(\theta)$ and the molecular axis distribution $\rho(\theta, t)$, where $\theta$ is the angle between the molecular axis and the laser polarization direction as in Eq. (3).

$$
Y(t)=\int \rho(\theta, t) R(\theta) \mathrm{d} \Omega
$$

The unknown angle dependence $R(\theta)$ is expanded in the Legendre polynomial basis, where $J$ can only take even values due to the plane of symmetry perpendicular to the polarization vector.

$$
R(\theta)=\sum_{J=0}^{J_{\max }} C_{J} P_{J}(\cos \theta)
$$

Mathematically, the expansion can go up to $J \rightarrow \infty$ as long as all the coefficients $C_{J}$ are determined (which can be 0 ). In practice, the expansion must be truncated at some value $J_{\max }$ since typically the description of $R(\theta)$ does not require $J \rightarrow \infty$, and we also do not have the ability to resolve an infinite number of coefficients. This $J_{\max }$ is determined by the function $R(\theta)$ and the high order coherences in the rotational wavepacket. In general, the higher the maximum alignment of the moleucles, the larger the value of $J_{\max }$ that can be kept in the expansion.

Substituting Eq. (4) into Eq. (3), we have

$$
Y(t)=\sum_{J=0}^{J_{\max }} C_{J}\left\langle P_{J}(\cos \theta)\right\rangle(t),
$$

where

$$
\left\langle P_{J}(\cos \theta)\right\rangle(t)=\int \rho(\theta, t) P_{J}(\cos \theta) \mathrm{d} \Omega .
$$

Since the delay $t$ and Legendre polynomial index $J$ take a finite number of values, Eq. (5) is a matrix equation that can be solved using linear regression methods [52] for $C_{J}$ (thus determining $\left.R(\theta)\right)$ if the $\left\langle P_{J}(\cos \theta)\right\rangle(t)$ matrix is known. The matrix is determined by the timedependent molecular axis distribution, which in turn depends on the rotational wavepacket launched by the pump pulse. In impulsive alignment, the rotational wavepacket (which determines the time-dependent molecular axis distribution) only depends on the laser fluence and 
the gas rotational temperature [53]. Hence, if the laser fluence and the rotational temperature can be measured accurately, the time evolution of the molecular axis distribution can be determined by solving the TDSE for rigidrotor (assuming the aligning pulse does not excite any vibrational or electronic states). However, in practice, laser fluence and rotational temperature measurements are not very accurate. Therefore, we use the measured values as an initial guess and fit the data $Y(t)$ over a grid of different pump laser intensities, pulse durations and gas rotational temperatures near the measured values. We consider the one that is best fitted to our data as the wave-packet that we have in the experiment. For the method to work, a long, high-quality delay scan with a high degree of alignment and a reliable fitting procedure are required $[34,54]$.

\section{B. Strong-field ionization of $\mathrm{CO}_{2}$ by linearly polarized pulses}

An example of the experimental data and fit to the $\mathrm{CO}_{2}^{+}$single ionization yield as function of delay probed by a $35 \mathrm{fs}, 140 \mathrm{TW} / \mathrm{cm}^{2}$ linearly polarized pulse is shown in Fig. 2. The fit with $J_{\max }=4$ according to the expansion in Eq. (4) shows excellent agreement with data. After the first aligning pulse $\left(250 \mathrm{fs}, 4.7 \mathrm{TW} / \mathrm{cm}^{2}\right)$, the molecular sample reaches a degree of alignment of $\left\langle\cos ^{2} \theta\right\rangle \approx 0.73$ (at half-revival), and after the second aligning pulse $\left(100 \mathrm{fs}, 14 \mathrm{TW} / \mathrm{cm}^{2}\right)$, the degree of alignment is improved to $\left\langle\cos ^{2} \theta\right\rangle \approx 0.86$. The rotational temperature of the gas is estimated to be about $2 \mathrm{~K}$ by the fit.

We retrieved the angle-dependent single-ionization probability from the delay-dependent data before and after the second aligning pulse in Fig. 2 separately to study how the retrieval depends on the degree of alignment. Fig. 3 shows the retrieved angle-dependent ionization probability and how it changes with different truncation of $R(\theta)$ in Eq. (4). We will denote it as $R^{\operatorname{lin}}(\theta)$ with the added superscript indicates linearly polarization of the probe. In panel (a), with high degree of alignment $\left\langle\cos ^{2} \theta\right\rangle \approx 0.86$, the high order $C_{J}$ coefficients can be determined reliably to be small and have little contribution to the retrieved angle-dependent ionization probability $R^{\operatorname{lin}}(\theta)$. In other words, $R^{\operatorname{lin}}(\theta)$ converges from $J_{\max }=4$. In panel (b), with $\left\langle\cos ^{2} \theta\right\rangle \approx 0.73$, the higher coefficients are less reliable, and the retrieved angle-dependent ionization probability $R^{\operatorname{lin}}(\theta)$ varies wildly with the expansion. Although the fit to the delay-dependent data converges in both cases, the extracted angular distribution in the case of lower degree of alignment is inconsistent since there can be more parameters in the fitting function than we can reliably determine.

We also performed angle-scan measurements [17] where the ionization yield $Y(\alpha)$ was measured as a function of angle $\alpha$ between polarization axes of the aligning pump pulses and the ionizing probe pulse. For these mea-
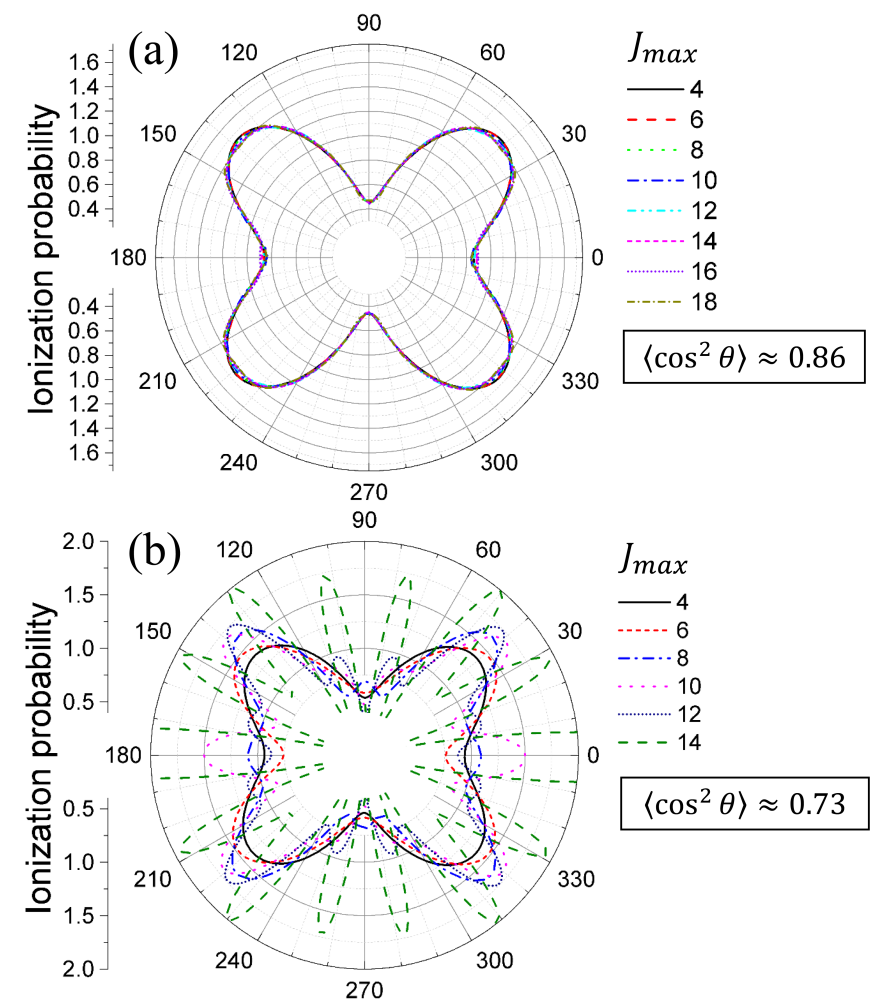

FIG. 3. Angle-dependent single-ionization probability of $\mathrm{CO}_{2}$ retrieved from the delay-dependent data in Fig. 2 for two different degrees of alignment and different orders of the expansion in Legendre polynomials according to Eq. (4). With $\left\langle\cos ^{2} \theta\right\rangle \approx 0.86$ (panel a), the high order coefficients can be determined reliably to be small and have little contribution to the retrieved angle-dependent ionization probability $R^{\operatorname{lin}}(\theta)$. With $\left\langle\cos ^{2} \theta\right\rangle \approx 0.73$ (panel b), the higher coefficients are less reliable, and the retrieved angle-dependent ionization probability $R^{\operatorname{lin}}(\theta)$ varies wildly with the expansion.

surements, the pump-pulse polarization axis is fixed but probe-pulse polarization is rotated by a half-wave plate mounted on a computer-controlled motorized rotational stage. In case of high degree of alignment, the molecular axis distribution will be narrow, which makes $Y(\alpha)$ a fair representation of $R^{\operatorname{lin}}(\theta)$.

The raw data is shown in Fig. 4(b) without using any deconvolution or fitting procedure. The error bars in the ionization yields represent the standard deviation from results of repeated experimental scans under the same condition. As expected from a higher degree of molecular alignment, our $Y(\alpha)$ have sharper features compared to previous data $[17,21]$ (which did not show a clear dip at $0^{\circ}$ ) and can be considered as a better representation of $R^{\operatorname{lin}}(\theta)$. Although $R^{\operatorname{lin}}(\theta)$ is sharper than $Y(\alpha)$ since the molecular distribution is deconcolved, they both have the same basic structure.

Despite having a higher degree of molecular alignment as compared to previous experiments [17, 20, 21], our deconvolved angle-dependent ionization probability $R^{\operatorname{lin}}(\theta)$ looks broader, with a peak around $40^{\circ}$. Measure- 


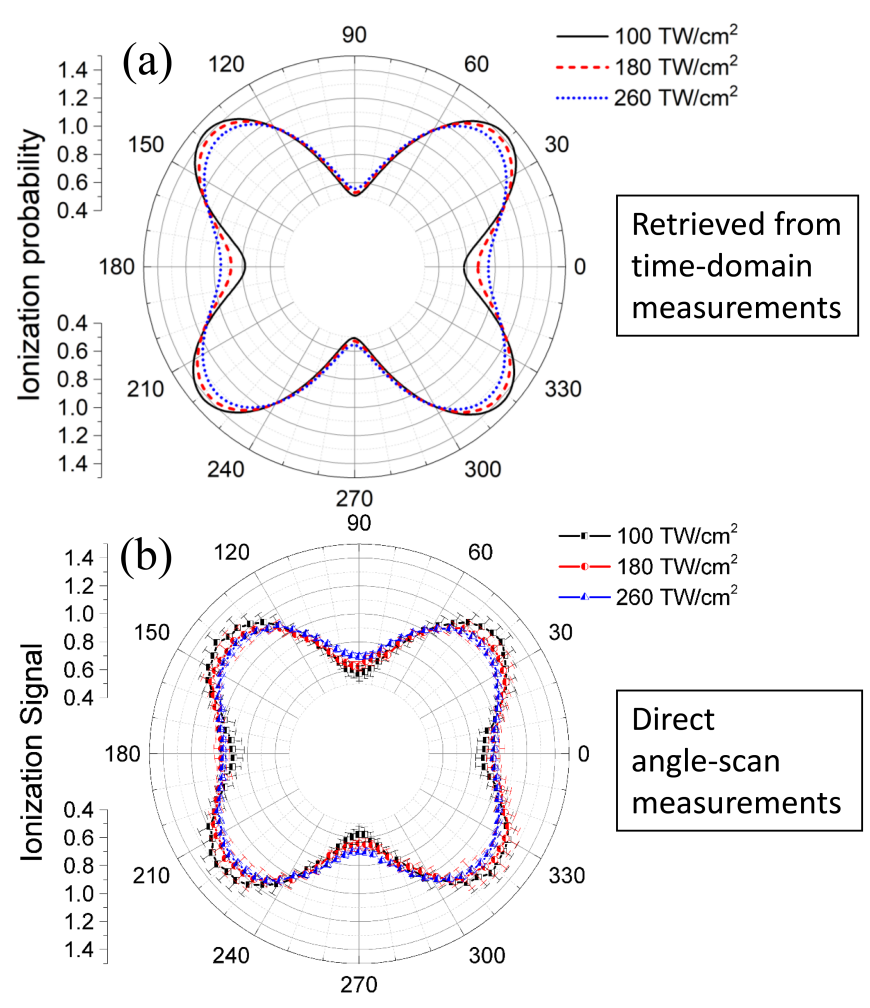

FIG. 4. Panel (a) shows the angle-dependent ionization probability $R^{\text {lin }}(\theta)$ retrieved from time-domain measurements for different probe intensities. The estimated degree of molecular alignment is $\left\langle\cos ^{2} \theta\right\rangle \approx 0.87$ in all cases. The expansion in Eq. (4) was truncated at $J_{\max }=6$. Using the same pump pulses, panel (b) shows measured ionization yield $Y(\alpha)$ as a function of angle $\alpha$ between polarization axes of the aligning pump pulses and the ionizing probe pulse at peak alignment (42.5 ps after the second pump) with no deconvolution or fitting. While $R^{\text {lin }}(\theta)$ is four-fold symmetric, $Y(\alpha)$ was measured over $2 \pi$ and has not been symmetrized.

ments at different intensities (see Fig. 4(a)) show that the angle-dependent ionization probability is more isotropic at higher probe intensities. Murray et al. theoretically predicted that the exact location of the maximum depends on the laser intensity [26]; however, this variation is too small in our experiment to make any solid conclusions.

\section{Strong-field ionization of $\mathrm{CO}_{2}$ by circularly polarized pulses}

The ORRCS approach is easily adapted to circularly polarized probes if the axial symmetry required for Eq. (4) is maintained by making the pump pulses circularly polarized as well. For this polarization geometry and multi-cycle laser pulses, the axis of symmetry is the laser propagation direction rather than the polarization direction. In order to avoid confusion with the linearly polarized case, we use the symbol $\beta$ for the polar angle between the molecular axis $\vec{m}$ and the propagation vector $\vec{k}_{p}$. When $\beta=0^{\circ}$, the molecules are along $\vec{k}_{p}$, we have kalignment; when $\beta=90^{\circ}$, the molecules are in the plane perpendicular to $\vec{k}_{p}$, and we have planar alignment [55].

Fig. 6 shows an example of experimental data and fit to $\mathrm{CO}_{2}^{+}$ionization yield by a circularly polarized probe as function of delay (a) and the retrieved angle-dependent ionization probability $R^{\text {cir }}(\beta)$ at different probe intensities (b). $R^{\text {cir }}(\beta)$ is closer to dumbbell shape in comparison with $R^{\text {lin }}(\theta)$ of butterfly shape. $R^{\text {cir }}(\beta)$ also becomes more isotropic at higher probe intensity.

Within the assumption of adiabatic ionization, we can perform a geometrical transformation from the angledependent ionization signal by the linearly polarized probe pulse $R^{\operatorname{lin}}(\theta)$ to re-produce the main features of the circular polarization measurements $R^{\operatorname{cir}}(\beta)[13,31]$. For a particular angle $\beta, R^{\text {cir }}(\beta)$ can be obtained by averaging $R^{\operatorname{lin}}(\theta)$ over all the electric field vectors sweeping the plane perpendicular to $\vec{k}_{p}$ (see Fig. 5). The molecular axis and the electric field can be written as $\vec{m}(0, \sin \beta, \cos \beta)$ and $\vec{E}(\cos \phi, \sin \phi, 0)$, then $\cos \theta=\vec{m} \cdot \vec{E}=\sin \beta \sin \phi$. $R^{\text {cir }}(\beta)$ will then be obtained by replacing $\cos \theta$ in the associated Legendre polynomial expansion of $R^{\operatorname{lin}}(\theta)$ in Eq. (4) by $\sin \beta \sin \phi$ and taking the average over $\phi$. The results in Fig. 6 show qualitative consistency between the two independent measurements using linearly and circularly polarized light.

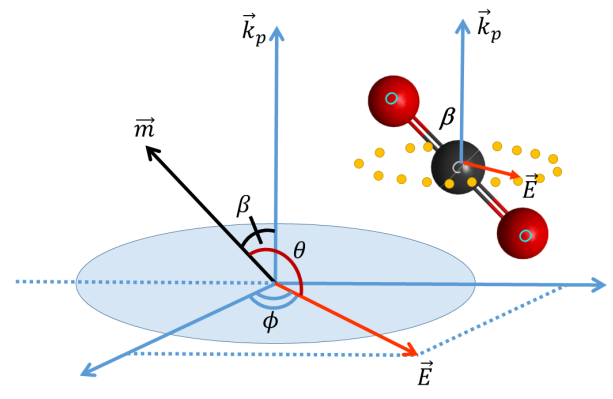

FIG. 5. Definition of angles: $\theta$ is the angle between the molecular axis, $\vec{m}$, and polarization axis. $\beta$ is the angle between the molecular axis and the electric field propagation vector, $\vec{k}_{p}$. Within the assumption of adiabatic ionization, the angledependent ionization probability, $R^{\text {cir }}(\beta)$, by a circularly polarized ionizing pulse can be obtained by averaging the angledependent ionization probability, $R^{\text {lin }}(\theta)$, by a linearly polarized ionizing pulse over the plane perpendicular to $\vec{k}_{p}$ (using angle $\phi$ ).

\section{ANGLE DEPENDENCE OF STRONG-FIELD MOLECULAR FRAGMENTATION}

\section{A. Introduction}

In the previous sections, we discussed non-dissociative single ionization by linear and circularly polarized pulses; 

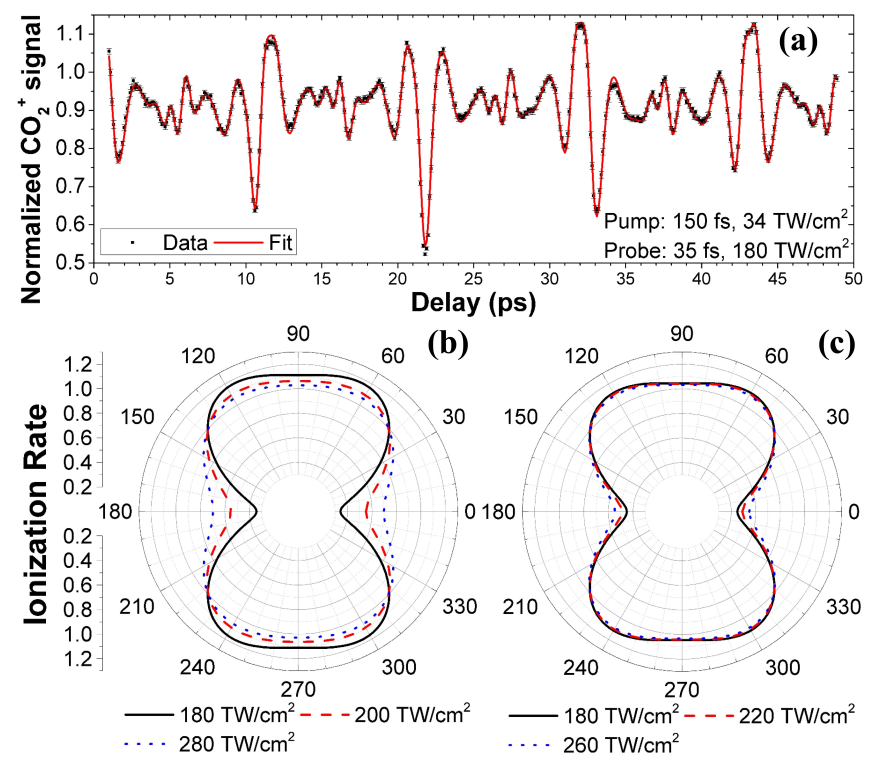

FIG. 6. (a) Experimental data and fit to $\mathrm{CO}_{2}^{+}$ionization yield by a circularly polarized probe as function of delay. These measurements used a single alignment pulse and achieved the degree of alignment $\left\langle\cos ^{2} \theta\right\rangle \approx 0.82$. At this degree of alignment, the convergence of $R^{\text {cir }}(\beta)$ in the same manner as in Fig. 3 from $J_{\max }=4$ was observed. (b) The retrieved angledependent ionization probability $R^{\text {cir }}(\theta)$ for different intensities. (c) Ionization probability $R^{\text {cir }}(\beta)$ by circularly polarized ionizing pulse obtained from the transformation of $R^{\operatorname{lin}}(\theta)$ measured with linearly polarized pulses in Sec. III B.

in this section, we shift our attention to dissociative double ionization by linearly polarized light. In dissociative ionization, not only the ionization probability, but also the angular distribution of the ions carries useful information. For example, the experimentally measured asymptotic momentum distribution of the fragments can be used to reconstruct the molecular axis distribution. However, this reconstruction can be hampered by the angledependent ionization probability (probe selectivity) and non-axial recoil [56-59]. Fig. 7 shows the directions of the molecular axis and the asymptotic momentum of the fragment ions and the definitions of the angles used in the following discussion. The molecular axis is shown at $\phi=0^{\circ}$; due to axial symmetry of the laser field along the polarization axis (linearly polarized light), the value of $\phi$ is not physically relevant, but we do lose the relevant angle $\phi_{k}-\phi$ due to this symmetry in the measurement. The molecular axis distribution can be obtained directly from the momentum distribution only if the vectors $\vec{m}$ and $\vec{k}$ coincide.

In this section, we demonstrate an experimental method to check whether molecular fragmentation by ultrafast pulses satisfies the axial recoil approximation. The basic idea is that we measure the angular distribution of the fragments as a function of the pump-probe delay, and compare it with the distribution of the molecular axis retrieved from the time evolution of the momentum

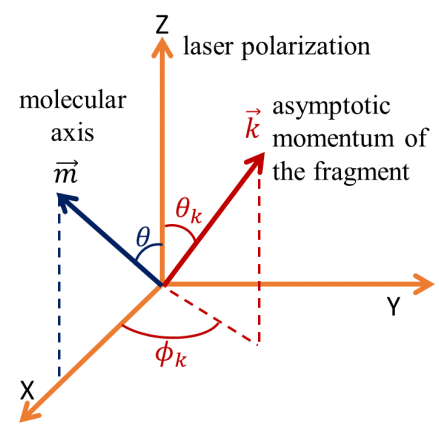

FIG. 7. Definition of angles describing the orientation of molecules (polar angle $\theta$ and azimuthal angle $\phi$ ) and asymptotic momentum of the fragments (polar angle $\theta_{k}$ and azimuthal angle $\phi_{k}$ ); $\mathrm{Z}$ defines the polarization axis in LF. The molecular axis distribution can be obtained directly from the momentum distribution only if the vectors $\vec{m}$ and $\vec{k}$ coincide. As mentioned in the text, the value of $\phi$ is not physically relevant, and the relevant angle $\phi_{k}-\phi$ is lost, the molecular axis is shown here at an arbitrarily chosen angle $\phi=0^{\circ}$.

distribution. Making this comparison for many different distributions from the time-evolution of the rotational wave-packet, we generate a probability distribution function that directly reflects the validity of the axial recoil approximation (the mathematical description is given in section IV C). Due to the loss of $\phi_{k}-\phi$ information as discussed above, $S(\vec{m}, \vec{k})=S\left(\theta, k, \theta_{k}\right)$. The probe selectivity (angle-dependent probability of the production of the fragment by the probe, or angle-dependent probability of dissociative double ionization) is also obtained simultaneously from the delay dependence of the fragment yield in the same manner as we analyze angle-dependent ionization, and is accounted for in the analysis. As an example, we discuss the application of this method to the fragmentation of $\mathrm{CO}_{2}$ by an intense, $35 \mathrm{fs}, 785 \mathrm{~nm}$ pulse in the following sections.

\section{B. Tpx3Cam and timing synchronization}

The experiment is also performed in a pump-probe arrangement where $\mathrm{CO}_{2}$ molecules are impulsively aligned by a non-resonant pump and then doubly ionized and dissociated by a probe pulse in a VMI experiment. In this case, we measure the LF $2 \mathrm{D}$ angular distribution of the fragment ions $M_{2 D}\left(\vec{k}_{2 D}, t\right)$ by using the time-stamping pixel camera Tpx3Cam to read out the signal created by the MCP and fast phosphor screen.

The Tpx3Cam is a hybrid pixel detector: an optical sensor with high quantum efficiency $[60,61]$ is bumpbonded to a Timepix3 ASIC [62], a time-stamping readout chip with $256 \times 256$ pixels, each with a size of $55 \mu \mathrm{m} \times 55 \mu \mathrm{m}$. The processing electronics in each pixel records the ToA (time of arrival) of hits that cross a preset threshold with ns resolution and stores it as timecode in a memory inside the pixel. The information about ToT 
(time-over-threshold), related to the energy deposited in each pixel, is also stored. The readout is data-driven with only $\mathrm{T}=475 \mathrm{~ns}+$ TOT pixel dead time, which allows multi-hit functionality at the pixel level and fast (80 Mpix/sec) throughput [63]. Tpx3Cam can also accept and time stamp an external trigger pulse, independent of the Timepix3 pixels. The granularity of the trigger time measurement is $0.26 \mathrm{~ns}$.

The Tpx3Cam camera allows us to simultaneously measure all of the fragment ions, and to synchronize single-shot data to external triggers for gas jet and pump pulse reliably, which is non-trivial at $2 \mathrm{kHz}$. In principle, 3D momentum distributions for ions can also be measured directly due to the $1.56 \mathrm{~ns}$ time resolution [64]. In the case of linearly polarized pulses, as we consider in this paper, using either the $2 \mathrm{D}$ or the $3 \mathrm{D}$ momentum distribution should lead to the same result apart from the complication of the data analysis.

The Tpx3Cam records the arrival time of trigger pulses obtained from the laser together with information of hits (coordinates, ToA, ToT). All other timing information about when the stage is in motion, whether the gas jet is on, and whether the optical chopper is allowing or blocking laser pulses was independently recorded by a PCIe6353 NI DAQ card. In order to correlate Tpx3Cam data with the timing information for every shot, the laser pulse trigger supplied to Tpx3Cam was disabled whenever the stage was in motion. The locations and durations of these missing triggers in the Tpx3Cam data provided enough information to correlate and sort the single-shot VMI data with the digital timing information from the DAQ card.

The performance of single-shot trigger status identification can be described as hits-per-shot histograms in Fig. 8(a). The number of hits here is from the whole spectrum, including residual gas which is mostly water. The histogram for each type of trigger should be a smooth one-peak distribution, any mislabeled hits will pile up as a second peak at the wrong place. If we consider all the hits in the left-peak of jet-on signals to be mislabeled and over-estimate it by a linear drop (in number of shots versus number of hits per shot), the result shows over four orders of magnitude of accuracy.

Fig. 8(b) depicts the total counts of $\mathrm{CO}^{+}$for each type of trigger, showing a good signal-to-noise ratio. When the gas jet is on, the number of hits (black solid line and red dotted line) is about 100 times more than that when the gas jet is off (blue dashed line and magenta dasheddotted line). The signal with aligning pulse (black line) exhibits a strong delay dependence while the modulation of the signal without the aligning pulse (due to fluctuation in laser intensity and gas density, indicated by the red dotted line) is much weaker.
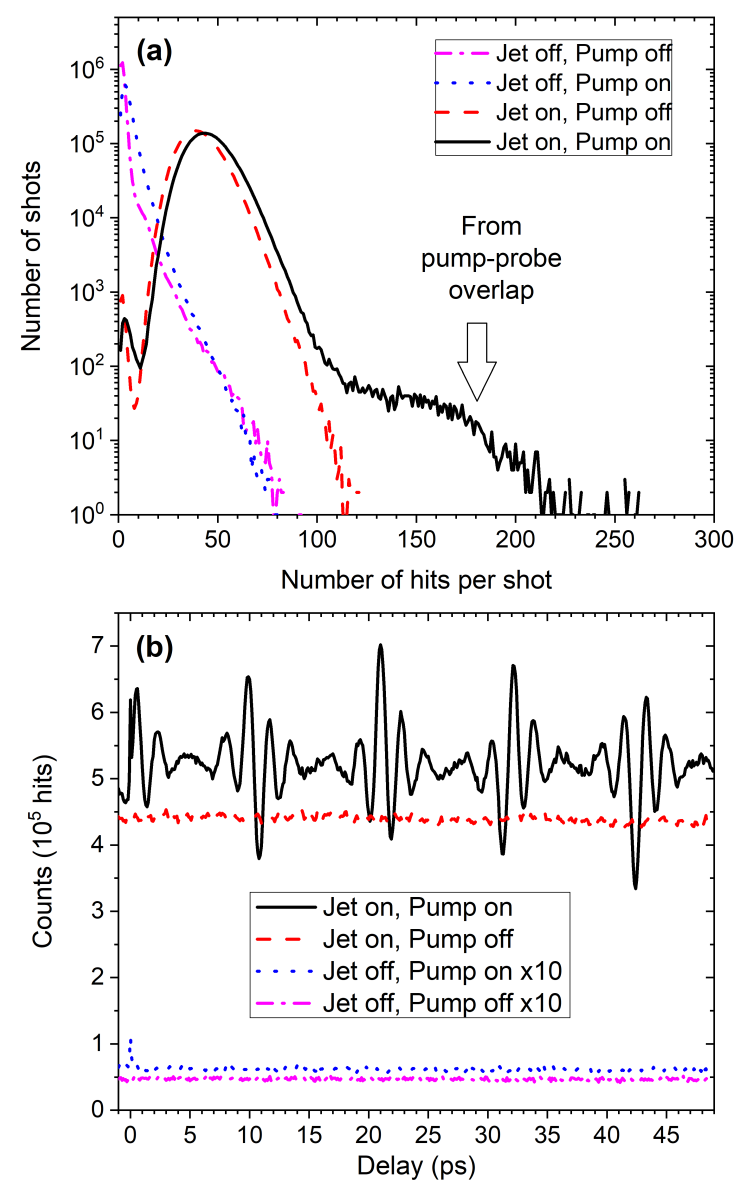

FIG. 8. (a) Hits-per-shot histograms for different trigger types of pump-probe-gas combination. (b) Total $\mathrm{CO}^{+}$counts for each type of trigger.

\section{Time-domain approach using ORRCS}

Our goal is to determine the probability distribution $S\left(\theta, k, \theta_{k}\right)$ of the probe pulse producing a fragment ion with momentum $k$ in the direction $\theta_{k}$ from molecule at angle $\theta$. To facilitate the determination of this function from experimental data, we express the angular dependence in a basis of the product of two Legendre polynomials $P_{J}(\cos \theta) P_{L}\left(\cos \theta_{k}\right)$ and the magnitude of the momentum in a discrete Gaussian basis as

$$
S\left(\theta, k, \theta_{k}\right)=\sum_{k_{0}, J, L} A_{k_{0} J L} \mathrm{e}^{-\frac{\left(k-k_{0}\right)^{2}}{2 \sigma^{2}}} P_{J}(\cos \theta) P_{L}\left(\cos \theta_{k}\right),
$$

where $k_{0}$ are the centers of the radial Gaussian functions, and $\sigma$ is the width of each of these functions. This choice of basis was motivated by the pBasex algorithm [65] that we use to analyze VMI data.

The delay-dependent momentum distribution of the fragment ion in the $\mathrm{LF}, M\left(k, \theta_{k}, t\right)$, is the convolution $S\left(\theta, k, \theta_{k}\right)$ and the delay-dependent molecular axis dis- 
tribution $\rho(\theta, t)$

$$
M\left(k, \theta_{k}, t\right)=2 \pi \int \rho(\theta, t) S\left(\theta, k, \theta_{k}\right) \sin \theta d \theta .
$$

Substituting Eq. (7) into Eq. (8), we have

$$
\begin{aligned}
M\left(k, \theta_{k}, t\right)=2 \pi \sum_{k_{0}, J, L} & {\left[A_{k_{0} J L} e^{-\frac{\left(k-k_{0}\right)^{2}}{2 \sigma^{2}}}\right.} \\
& \left.\times\left\langle P_{J}(\cos \theta)\right\rangle(t) P_{L}\left(\cos \theta_{k}\right)\right] .
\end{aligned}
$$

where $\left\langle P_{J}(\cos \theta)\right\rangle(t)=\int \rho(\theta, t) P_{J}(\cos \theta) \sin \theta \mathrm{d} \theta$ are delay-dependent axis distribution moments.

In this experiment, we do not measure $M\left(k, \theta_{k}, t\right)$ directly but only the $2 \mathrm{D}$ projection of the charged sphere on the VMI detector at each delay. Due to the axial symmetry of the momentum distribution, we can reconstruct $M\left(k, \theta_{k}, t\right)$ from the data by applying the pBasex method [65] at each delay to obtain a set of delay-dependent coefficients $C_{k_{0} L}(t)$ that represent $M\left(k, \theta_{k}, t\right)$ as

$$
M\left(k, \theta_{k}, t\right)=2 \pi \sum_{k_{0}, L} C_{k_{0} L}(t) e^{-\frac{\left(k-k_{0}\right)^{2}}{2 \sigma_{b}^{2}}} P_{L}\left(\cos \theta_{k}\right) .
$$

Comparing Eqs. (9) and (10), we get

$$
C_{k_{0} L}(t)=\sum_{J} A_{k_{0} J L}\left\langle P_{J}(\cos \theta)\right\rangle(t)
$$

which is identical in form to Eq.(5), and can be solved in the same manner by linear regression. One important difference is that Eq.(11) is a large set of equations (one for each pair of $k_{0}$ and $L$ ), which allows for additional consistency checks since all the fits should converge to the same rotational wavepacket parameters (pump fluence and rotational temperature).

In the measurements, a channel usually spans a finite range of the radial momentum $k$ (radial width), so we need to integrate over that range of $k$ for each channel. Usually, over the spanned range of $k$, the channel has the same angular distribution and the integration simply increases the statistics. On the other hand, if the fragment angular distribution behaves differently within the energy spectrum, this indicates that a measurement with higher energy-resolution should be done to finely resolve all the involved states or channels.

The fragment angular distribution of a single channel in the LF can also be described in terms of the widelyused asymmetry parameters, $\beta_{L},[66]$ as

$$
M\left(k, \theta_{k}, t\right)=\frac{\sigma(k, t)}{4 \pi}\left[1+\sum_{\text {even } \mathrm{L}} \beta_{L}(k, t) P_{L}\left(\cos \theta_{k}\right)\right]
$$

where $\sigma(k, t)$ is the total (angles $\theta_{k}$ and $\theta$ integrated) fragmentation cross section; the time dependence comes from the dependence on the molecular axis distribution $\rho(\theta, t)$. Putting Eq. (10) and Eq. (12) in the same form, the asymmetry parameters - averaged over the range of $k$ for the channel - can be written as

$$
\beta_{L}(\bar{k}, t)=\frac{C_{\bar{k} L}(t)}{C_{\bar{k} 0}(t)}
$$

and the total angle-integrated cross section is $\sigma(\bar{k}, t)=$ $8 \pi^{2} C_{\bar{k} 0}(t)$.

\section{D. $\mathrm{CO}^{+}$and $\mathrm{O}^{+}$fragments at $1 \times 10^{15} \mathrm{~W} / \mathrm{cm}^{2}$}

In this section, we apply the analysis discussed in section IV C to fragmentation of $\mathrm{CO}_{2}$ by an intense, $35 \mathrm{fs}$, $785 \mathrm{~nm}$ pulse with data acquired by the method described in section IV B. At a probe-pulse intensity of $1 \times 10^{15}$ $\mathrm{W} / \mathrm{cm}^{2}, \mathrm{CO}_{2}$ molecules can be doubly ionized and dissociate into $\mathrm{CO}^{+}$and $\mathrm{O}^{+}$fragments. The ions corresponding to this channel can be discriminated from other channels in the VMI images using momentum and energy conservation, assuming a Coulomb explosion. This identification, shown in Fig. 9, is in good agreement with data from coincidence measurements [67].
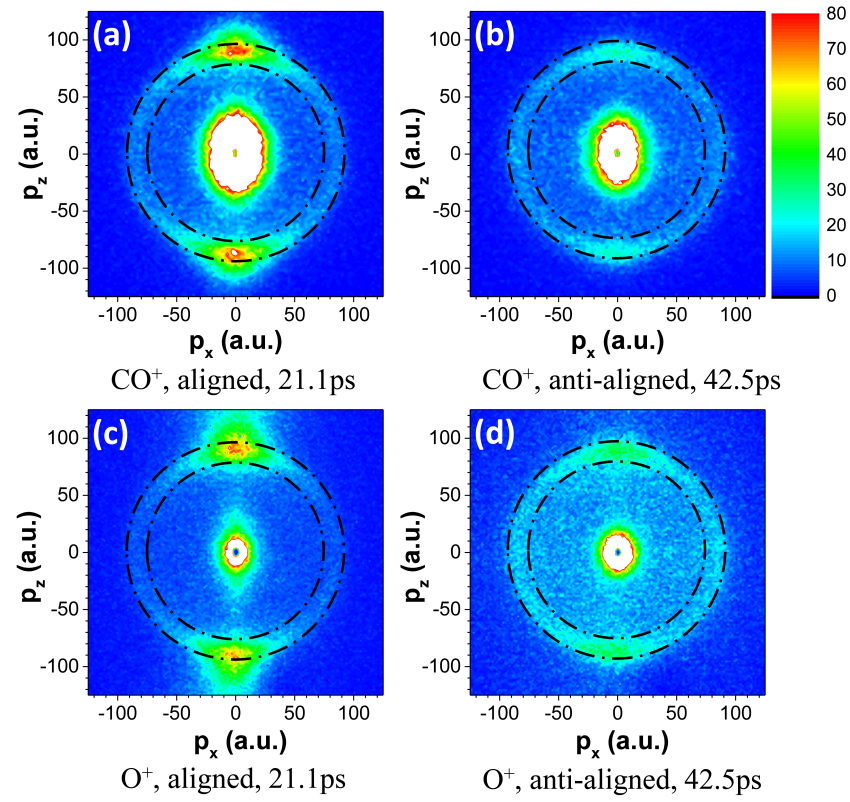

FIG. 9. Raw VMI images of $\mathrm{CO}^{+}$and $\mathrm{O}+$ at the aligned $(\mathrm{a}, \mathrm{c})$ and anti-aligned peaks $(\mathrm{b}, \mathrm{d})$. The laser polarization is along the $p_{z}$ direction. The linear color scale expresses the yield of the ions in arbitrary units.. The circles indicate the regions of interests corresponding to the fragmentation of $\mathrm{CO}_{2}^{2+}$ into two singly charged ions (note that a specific channel was selected from the inverted data, not on the raw data). Fragments with low energy coming from singly charged molecular ion are saturated on the color map for display purposes.

In our data, each fragment ion can be selected by putting a gate of $300 \mathrm{~ns}$ on the recorded ToA. By doing so, we mostly look at the prompt breakup, but there can still be some contribution from long-lived $\mathrm{CO}_{2}^{2+}[67]$. The 


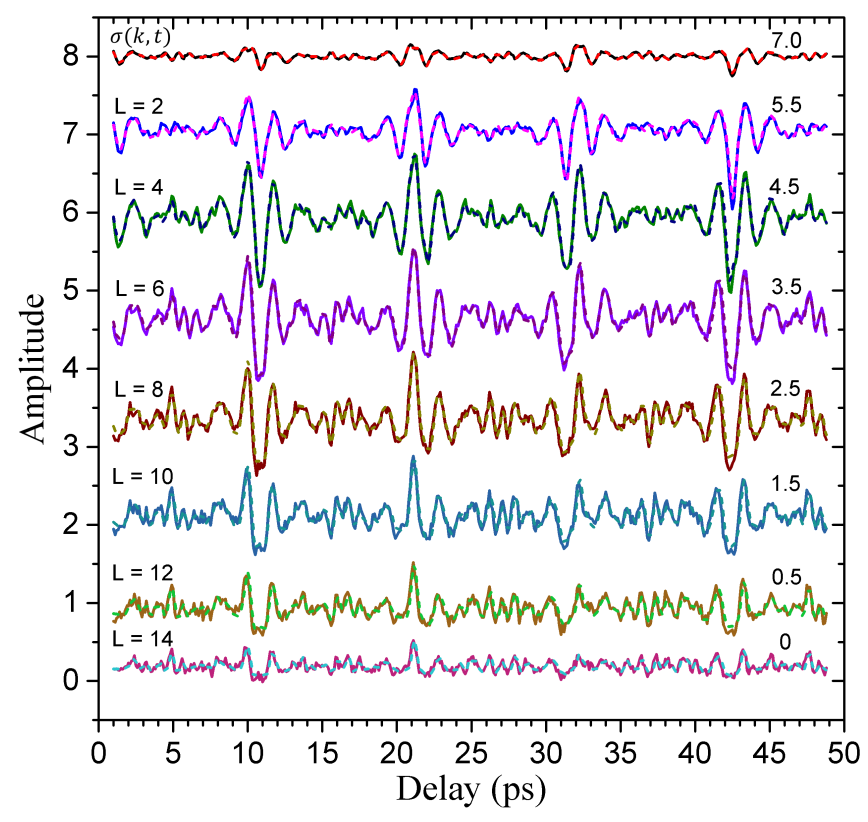

FIG. 10. Time evolution of the asymmetry parameters $\beta_{L}(k, t)$ (solid) and the angle-integrated cross section $\sigma(k, t)$ together with their corresponding fits (dashed) for $\mathrm{CO}^{+}$fragment at a probe intensity of $1 \times 10^{15} \mathrm{~W} / \mathrm{cm}^{2}$. The aligning pump pulse in this case is about $150 \mathrm{fs}, 20 \mathrm{TW} / \mathrm{cm}^{2}$. For display purpose, $\sigma(k, t)$ is normalized by its mean. For clarity, each plot was shifted vertically by an amount indicated by the number on the right side.

raw VMI images in Fig. 9 show the asymptotic momentum spectra of $\mathrm{CO}^{+}$and $\mathrm{O}^{+}$at aligned and anti-aligned peaks. The circles indicate the regions of interests in which the fragments were analyzed.

The time evolution of asymmetry parameters, $\beta_{L}(k, t)$, and the angle-integrated cross section, $\sigma(k, t)$, together with their corresponding fits for $\mathrm{CO}^{+}$fragment are shown in Fig. 10. We obtained excellent fits up to $L=14$ with $J_{\max }=14$, although the fits already converge from $J_{\max }=6\left(J_{\max }\right.$ determines the truncation of Eq. $\left.(7)\right)$.

A single alignment pump (150 fs, $20 \mathrm{TW} / \mathrm{cm}^{2}$ ) was used in this measurement. The fluence of the aligning pulse and the rotational temperature of the gas (around $2 \mathrm{~K})$ are retrieved consistently from fits with different $\beta_{L}(k, t)$ and $\sigma(k, t)$ for both $\mathrm{O}^{+}$and $\mathrm{CO}^{+}$fragments as seen in Fig. 11. From these fits, we get $A_{k_{0} J L}$ coefficients and determine the angle-dependent probability $S\left(\theta, k, \theta_{k}\right)$; the retrieved function is shown in Fig. 12(b).

While the asymmetry parameters $\beta_{L}(k, t)$ (and $A_{k_{0} J L}$ coefficients) determine the angular distribution in the LF (and MF), the probe selectivity $F(\theta)$ is also obtained simultaneously in the measurement by fitting to the time evolution of the angle-integrated cross section $\sigma(k, t)$. $F(\theta)$, which can also be interpreted as the fragmentation rate, is the probability of making a given fragment as a function of molecular orientation $(\theta)$ in the laser field as shown in Fig. 12(a). $F(\theta)$ can also be obtained by integrating $S\left(\theta, k, \theta_{k}\right)$ over $\theta_{k}$. Both methods give consistent

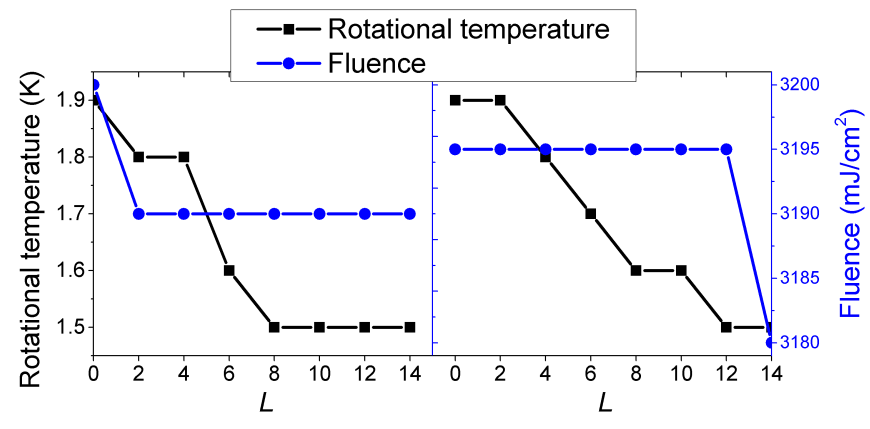

FIG. 11. The fluence of the aligning pulse and the rotational temperature of the gas, which determine the rotational wave-packet, are retrieved consistently from fits with different asymmetry parameters $\beta_{L}(k, t)$ for both $\mathrm{O}^{+}$(left panel) and $\mathrm{CO}^{+}$(right panel) fragments. This indicates the reliability of the retrieval procedure. At the value of $L=0$, since $\beta_{0}$ is always 1 , we are showing parameters retrieved from the fit on the cross-section $\sigma(k, t)$ instead.

results.

We can now correct for the probe selectivity by normalizing each vertical line of $S\left(\theta, k, \theta_{k}\right)$ by $F(\theta)$. The new function, $D\left(k, \theta_{k} \mid \theta\right)$, shown in Fig. 12(c) expresses the conditional probability for a fragment to fly out at $\theta_{k}$ given that the fragment is created from a molecule that was aligned along $\theta$ prior to the probe pulse. In this case, since $F(\theta)$ is not a very sharp function (with a maximum to minimum ratio of about 1.7$), D\left(k, \theta_{k} \mid \theta\right)$ enhances some features (as discussed below), but does not differ significantly from $S\left(\theta, k, \theta_{k}\right)$.

If the asymptotic momentum of the fragment is a good representation of the initial orientation of the neutral molecule, then $\theta_{k} \approx \theta$ and hence a diagonal band should be observed. However, in the $D\left(k, \theta_{k} \mid \theta\right)$ plot (Fig. 12(c)), there is only a very weak trend along the diagonal line while the major trend peaks along the laser polarization direction $\left(\theta_{k}=0\right)$ regardless of the initial orientation of the molecules. This clearly shows a breakdown of the axial recoil approximation (i.e., the asymptotic momentum angular distribution of the fragment does not reflect the molecular axis distribution before the probe pulse). The molecules are likely to undergo significant realignment during the process.

In our experiment, the estimated anti-alignment peak is sharp with $\left\langle\cos ^{2} \theta\right\rangle \approx 0.1$, so molecules are well confined near the plane perpendicular to the polarization axis (horizontal plane). Hence, the distribution as a line along the $p_{x}$ axis should be observed if the axial-recoil approximation is held strongly, which is not the case as seen from the raw images (Fig. 9). Using the retrieved $S\left(\theta, k, \theta_{k}\right)$, we estimate $\left\langle\cos ^{2} \theta_{k}\right\rangle$ of an isotropic distribution measured by this probe on this channel to be about 0.51 , in good agreement with the value of $0.506 \mathrm{calcu}-$ lated from the 3D distribution reconstructed by pBasex inversion.

As mentioned in Sec. IV B, the time-stamping pixel 

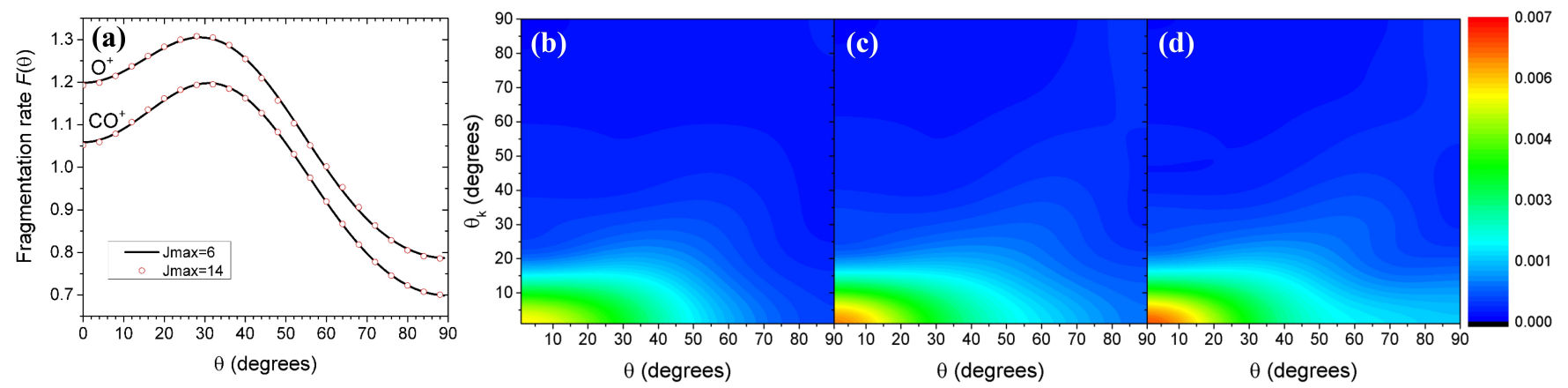

FIG. 12. (a) The fragmentation rate $F(\theta)$ expressing the probability of producing the fragment as a function of initial molecular orientation $\theta$ relative to the probability from an isotropic ensemble. For display purpose, $F(\theta)$ for $\mathrm{O}^{+}$is shifted up by 0.1 . (b) Angle-dependent probability $S\left(\theta, k, \theta_{k}\right)$ expressing how the angular distribution of the $\mathrm{CO}^{+}$fragment depends on the orientation of the molecule relative to the laser polarization. (c) Angle-dependent conditional probability $D\left(k, \theta_{k} \mid \theta\right)$ expressing the probability for a fragment to fly out at $\theta_{k}$ given that the fragment is created from a molecule that was aligned along $\theta$ prior to the probe pulse. (d) $D\left(k, \theta_{k} \mid \theta\right)$ plot for $\mathrm{O}^{+}$fragment coming from the same channel.

detector Tpx3Cam allows us to simultaneously measure all the fragment ions. Analysis of $\mathrm{O}^{+}$momenta measured simultaneously (but not in coincidence because the count rate was too high for coincident detection) shows very similar results (see Fig. 11 and Fig. 12).

It is worth noting that our experiment cannot distinguish whether the realignment happens during the (double-)ionization step $\left(\mathrm{CO}_{2} \rightarrow \mathrm{CO}_{2}^{2+}\right)$ or during the subsequent fragmentation of the dication $\left(\mathrm{CO}_{2}^{2+} \rightarrow\right.$ $\left.\mathrm{CO}^{+}+\mathrm{O}^{+}\right)$. The result is the total effect from neutral molecules to fragments $\left(\mathrm{CO}_{2} \rightarrow \mathrm{CO}_{2}^{2+} \rightarrow \mathrm{CO}^{+}+\mathrm{O}^{+}\right)$.

We would like to mention that this experiment is closely related to other works on imaging the rotational wave-packets using momentum measurements [68$70]$ since those measurements require the axial-recoil breakup of the molecules to a certain extent. While the probe selectivity (angle-dependent ionization probability) has been previously considered and minimized in those pump-probe arrangements, the realignment of molecules in the strong laser field was not discussed explicitly. This effect can be avoided by probing a subset of molecules where the torque exerted by the laser field is small [68] or choosing the observables that are independent of the realignment [70]. In our analysis, it is equivalent to looking at molecules near $\theta_{k} \approx 90^{\circ}$. In that vicinity, $\mathrm{CO}_{2}$ molecules also follow axial-recoil breakup to certain extent indicated by the local maxima (top-right corners) of the $D\left(k, \theta_{k} \mid \theta\right)$ plots in Fig. 12(c-d).

\section{CONCLUSIONS}

We have retrieved the angle dependence of the ionization probability of $\mathrm{CO}_{2}$ from time-domain measurements on impulsively-excited rotational wave-packets. The result is in good agreement with direct angle-scan measurement and is consistent with an independent experiment using a circularly polarized probe pulse. Although our experiment has shown high angular resolution, the angle- dependent ionization probability is mainly determined by the first three coefficients in Eq. (4). Coefficients with order higher than 4 were determined to be relatively small. This leads to a fair agreement of our final result with previous works at lower degrees of alignment by Thomann et al. [20] and Weber et al. [21] where Thomann et al. had used the expansion up to $J_{\max }=4$. The angle dependence of the ionization probability tends to have shallower peak-to-trough contrast at higher intensity while maintaining the overall shape.

The method shown here can, in principle, be used to characterize SFI at any wavelength. In particular, it would be useful to measure the angle-dependence of SFI using mid-IR lasers, including cases where the laser wavelength coincides with vibrational resonances. This information would be helpful for using LIED [4] to measure the molecular structure.

We have also developed a method to retrieve the probability function $S\left(\theta, k, \theta_{k}\right)$ that describes how the ejection of fragments depends on the orientation of the molecule. This method provides a quantitative check of the axialrecoil approximation. As demonstrated, the axial-recoil approximation does not hold for the $\mathrm{CO}_{2}^{2+} \rightarrow \mathrm{CO}^{+}+$ $\mathrm{O}^{+}$channel that we observed at $1 \times 10^{15^{2}} \mathrm{~W} / \mathrm{cm}^{2}$. The molecules are likely to undergo significant realignment during the ionization and fragmentation process. Although in these strong-field experiments, we cannot point out exactly when the realignment happens, the idea that we proposed would be very useful for experiments using a weak-field probe (say, x-ray) or short pulses since the re-alignment effect of the probe before ionization will be negligible. In that case, the result will be purely the angle dependence of the light-induced dynamical (ionization, fragmentation, etc.) processes. For $\mathrm{CO}_{2}$; however, short pulses $(<20 \mathrm{fs})$ can excite vibrations in the electronic ground state of the neutral via far-off-resonance two-photon Raman scattering [71].

In our axially symmetric measurements, only the polar angle dependence can be retrieved while the azimuthal 
angle is averaged. In order to complete the MF ion angular distributions, 3D momentum spectra without axial symmetry of the molecular axis distribution (by using elliptically polarized pump pulses for example) are required. Such information can be measured directly by using the Tpx3Cam due to the $1.56 \mathrm{~ns}$ time resolution of the detector (in combination with fast phosphor screen and a uniform field spectrometer) or it can be reconstructed using computed tomography [72].

Using the same analysis on a photoelectron momentum measurement, the photoelectron angular distribution in the MF would be possible to be retrieved.

The time-domain approach is not limited to molecular symmetry and laser polarization; when used to analyze ionization and fragmentation of more complex asymmetric top molecules, it can give the dependence on two Euler angles (polar angle $\theta$ and azimuthal angle in MF $\chi$ ) [34]. The momenta analysis does not require a charged partner and can be applied to dissociative ionization of singly charged ion. An extension of this method to pulses of different ellipticity can give more insights to understand processes triggered by re-collision $[13,21,38]$. This information usually cannot be obtained by other means.

\section{ACKNOWLEDGMENTS}

This work was supported by the U.S. Department of Energy, Office of Science, Basic Energy Sciences, under Awards \#DE-FG02-86ER13491 and \#DE-FG0208ER15984.
[1] X. Ren et al., Phys. Rev. A 88, 043421 (2013).

[2] A. Rupenyan et al., Phys. Rev. A 87, 033409 (2013).

[3] H. Timmers et al., Phys. Rev. Lett. 113, 113003 (2014).

[4] B. Wolter et al., Science 354, 308 (2016).

[5] A.-T. Le et al., Journal of Physics B: Atomic, Molecular and Optical Physics 49, 053001 (2016).

[6] L. He et al., Phys. Rev. Lett. 121, 163201 (2018).

[7] F. Krečinić et al., Phys. Rev. A 98, 041401 (2018).

[8] Y. He et al., Phys. Rev. A 99, 053419 (2019).

[9] A. Perelomov et al., JETP 23, 924 (1965).

[10] M. Ammosov et al., JETP 64, 1191 (1986).

[11] A. Becker and F. H. M. Faisal, Journal of Physics B: Atomic, Molecular and Optical Physics 38, R1 (2005).

[12] X. M. Tong et al., Phys. Rev. A 66, 033402 (2002).

[13] P. Hoerner and H. B. Schlegel, The Journal of Physical Chemistry A 121, 1336 (2017).

[14] P. Sándor et al., Phys. Rev. A 98, 043425 (2018).

[15] N. I. Shvetsov-Shilovski et al., Phys. Rev. A 98, 023406 (2018).

[16] A. I. Dnestryan et al., The Journal of Chemical Physics 149, 164107 (2018).

[17] D. Pavičić et al., Phys. Rev. Lett. 98, 243001 (2007).

[18] S. Petretti et al., Phys. Rev. Lett. 104, 223001 (2010).

[19] V. P. Majety and A. Scrinzi, Phys. Rev. Lett. 115, 103002 (2015).

[20] I. Thomann et al., The Journal of Physical Chemistry A 112, 9382 (2008).

[21] S. Weber et al., Journal of Modern Optics 60, 1379 (2013).

[22] S.-K. Son and S.-I. Chu, Phys. Rev. A 80, 011403 (2009).

[23] M. Abu-samha and L. B. Madsen, Phys. Rev. A 80, 023401 (2009).

[24] S.-F. Zhao et al., Phys. Rev. A 80, 051402 (2009).

[25] M. Spanner and S. Patchkovskii, Phys. Rev. A 80, 063411 (2009).

[26] R. Murray et al., Phys. Rev. Lett. 106, 173001 (2011).

[27] C. Wu et al., Phys. Rev. A 83, 033410 (2011).

[28] H. Shi-Lin et al., Chinese Physics Letters 30, 103103 (2013).

[29] J. Yao et al., Phys. Rev. Lett. 111, 133001 (2013).

[30] M. D. Śpiewanowski and L. B. Madsen, Phys. Rev. A 91, 043406 (2015).
[31] P. Krause and H. B. Schlegel, The Journal of Physical Chemistry Letters 6, 2140 (2015).

[32] V.-H. Hoang et al., Phys. Rev. A 95, 023407 (2017).

[33] C. Marceau et al., Journal of Physics B: Atomic, Molecular and Optical Physics 53, 084006 (2020).

[34] V. Makhija et al., "Orientation resolution through rotational coherence spectroscopy," (2016), arXiv: 1611.06476 .

[35] H. Stapelfeldt and T. Seideman, Rev. Mod. Phys. 75, 543 (2003).

[36] C. P. Koch et al., Rev. Mod. Phys. 91, 035005 (2019).

[37] I. Petersen et al., Phys. Rev. Lett. 114, 103004 (2015).

[38] D. Baykusheva and H. J. Wörner, Phys. Rev. X 8, 031060 (2018).

[39] R. Trebino et al., Review of Scientific Instruments 68, 3277 (1997).

[40] B. Schaefer et al., American Journal of Physics 75, 163 (2007).

[41] M. Hillenkamp et al., The Journal of Chemical Physics 118, 8699 (2003).

[42] A. T. J. B. Eppink and D. H. Parker, Review of Scientific Instruments 68, 3477 (1997).

[43] N. G. Kling et al., Journal of Instrumentation 9, P05005 (2014).

[44] A. Zhao et al., Review of Scientific Instruments 88, 113104 (2017).

[45] A. Nomerotski, Nuclear Instruments and Methods in Physics Research Section A: Accelerators, Spectrometers, Detectors and Associated Equipment 937, 26 (2019).

[46] J. Mikosch et al., Phys. Rev. Lett. 110, 023004 (2013).

[47] J. Mikosch et al., The Journal of Chemical Physics 139, 024304 (2013).

[48] P. Sándor et al., The Journal of Chemical Physics 151, 194308 (2019).

[49] C. Vozzi et al., Nature Physics 7, 822 (2011).

[50] C. Marceau et al., Phys. Rev. Lett. 119, 083401 (2017).

[51] L. S. Spector et al., Nature Communications 5, 3190 (2014).

[52] M. Kuhn and K. Johnson, Applied Predictive Modeling (Springer-Verlag New York, 2013).

[53] L. Holmegaard et al., Phys. Rev. A 75, 051403 (2007).

[54] X. Wang et al., Phys. Rev. A 96, 023424 (2017). 
[55] C. T. L. Smeenk et al., Phys. Rev. Lett. 112, 253001 (2014).

[56] J. G. Underwood et al., Review of Scientific Instruments 86, 073101 (2015).

[57] L. Christensen et al., Phys. Rev. A 94, 023410 (2016).

[58] D. Reedy et al., Phys. Rev. A 98, 053430 (2018).

[59] Z. L. Streeter et al., Phys. Rev. A 98, 053429 (2018).

[60] M. Fisher-Levine and A. Nomerotski, Journal of Instrumentation 11, C03016 (2016).

[61] A. Nomerotski et al., Journal of Instrumentation 12, C01017 (2017).

[62] T. Poikela et al., Journal of Instrumentation 9, C05013 (2014).

[63] B. van der Heijden et al., Journal of Instrumentation 12, C02040 (2017).

[64] M. Fisher-Levine et al., Journal of Synchrotron Radiation
25, 336 (2018).

[65] G. A. Garcia et al., Review of Scientific Instruments 75, 4989 (2004).

[66] R. N. Zare, Angular Momentum: Understanding Spatial Aspects in Chemistry and Physics (John Wiley \& Sons, Inc, 1988).

[67] H. Hu et al., "Laser-induced dissociative recombination of carbon dioxide," (2019), arXiv:1906.10429.

[68] P. W. Dooley et al., Phys. Rev. A 68, 023406 (2003).

[69] A. S. Mouritzen and K. Mølmer, The Journal of Chemical Physics 124, 244311 (2006).

[70] E. Karamatskos et al., Nature Communications 10, 3364 (2019).

[71] A. Rudenko et al., Faraday Discuss. 194, 463 (2016).

[72] J. Maurer et al., Phys. Rev. Lett. 109, 123001 (2012). 